Patrícia Honório Silva Santos ${ }^{1}$ Marcos Henrique Fernandes ${ }^{1}$ Cezar Augusto Casotti ${ }^{1}$ Raildo da Silva Coqueiro ${ }^{1}$ José Ailton Oliveira Carneiro ${ }^{1}$

${ }^{1}$ Departamento de Saúde, Universidade Estadual do Sudoeste da Bahia. Conjunto Urbis I, Jequiezinho. 45206510 Jequié BA Brasil. patyhonorios@hotmail.com

\section{Perfil de fragilidade e fatores associados em idosos cadastrados em uma Unidade de Saúde da Família}

\author{
The profile of fragility and associated factors \\ among the elderly registered in a Family Health Unit
}




\section{Introdução}

O envelhecimento populacional é um fenômeno mundial, o qual desencadeia mudanças no perfil das doenças que acometem esse grupo etário. Segundo Lourenço et al. ${ }^{1}$, 10 a $25 \%$ da população idosa é portadora de determinadas condições clínicas que os colocam em categorias de fragilidade, necessitando de cuidados intensivos e elevados custos para o governo e família.

O termo fragilidade tem surgido como um conceito muito importante em geriatria e gerontologia, aumentando na mesma proporção em que aumenta o número de idosos, e elevando o risco de eventos adversos à saúde como queda, incapacidades, institucionalização e mortalidade, repercutindo em impactos na vida dos idosos, seus familiares, cuidadores e toda a sociedade ${ }^{2}$.

No entanto, ainda não existe um consenso sobre a definição de fragilidade. Há cerca de 30 anos é reconhecida por diferentes autores, a existência de um quadro de fragilidade que atingia alguns idosos, cujo conceito vem se modificando com o passar do tempo. Inicialmente a fragilidade foi definida apenas pela natureza funcional, ou seja, era considerado frágil o idoso com variáveis graus de dependência ${ }^{3}$.

Na última década, a fragilidade tem sido definida como uma síndrome de declínio de energia, fundamentada em alterações que ocorrem com o envelhecimento, como sarcopenia, desregulação neuroendócrina e disfunção imunológica, que predispõe os idosos a redução acentuada da massa muscular e a um estado inflamatório crônico, que, caso associado a fatores extrínsecos de diversas naturezas, como doenças, imobilidade, diminuição da alimentação, entre outros, ocasiona um ciclo de redução de energia, aumentando a dependência e susceptibilidade a agressores, manifestando-se por velocidade de marcha reduzida, perda de peso, fadiga, diminuição da força de preensão e baixo nível de atividade física ${ }^{4}$.

Segundo Vieira et al. ${ }^{5}$, os idosos fragilizados são aqueles que apresentam maior número de fatores associados e podem estar relacionados com maior ocorrência de doenças crônicas, quedas, sintomas depressivos, limitações em atividades instrumentais da vida diária, redução da autoeficácia, hospitalização e idade mais avançada. Remor et al. ${ }^{6}$ ainda acrescentaram como fatores associados à fragilidade o tipo de moradia, inatividade e consumo de bebidas alcoólicas. Sendo assim, torna-se importante conhecer os fatores associados à fragilidade, para que medidas preventivas sejam adotadas no intuito de minimizar a síndrome e seus desfechos adversos, reduzindo o impacto na qualidade de vida da população idosa.

Diante do exposto, este estudo teve como objetivo identificar o perfil de fragilidade e os fatores associados em idosos cadastrados em uma Unidade de Saúde da Família.

\section{Métodos}

Trata-se de um estudo observacional de corte transversal, baseado em dados secundários extraídos do banco de dados da pesquisa intitulada "PET- Saúde Jequié: Identificando o processo saúde-doença em população adstrita às Unidades de Saúde da Família” o qual foi aprovado pelo Comitê de Ética em Pesquisa da Universidade Estadual do Sudoeste da Bahia. Teve como cenário o domić́lio dos idosos residentes no bairro Pau Ferro, do município de Jequié/BA, os quais são cadastrados na Unidade de Saúde da Família Giserlando Biondi.

Participaram do estudo 139 idosos com idade igual ou superior a 60 anos, capazes de responder ao questionário e realizar os testes de força de preensão manual, teste de equilíbrio, mobilidade e de caminhada. Aqueles idosos que não se adequaram a esses critérios foram excluídos da pesquisa.

Os dados foram coletados no período de maio a novembro de 2013, por doze monitores de diferentes áreas da saúde, após treinamento prévio, com supervisão de seis preceptores e um tutor do Programa de Educação pelo Trabalho para Saúde/PET-Saúde Grupo Idoso I, da Universidade Estadual do Sudoeste da Bahia. Foram respeitadas as diretrizes da resolução no 466 , de 12 de Dezembro de $2012^{7}$. Todos os participantes foram informados detalhadamente sobre os objetivos da pesquisa, procedimentos adotados e garantia de anonimato e sigilo dos dados referentes à sua identificação e de sua família. A participação dos mesmos foi voluntária, não tendo nenhum benefício econômico. Após esclarecimentos do estudo, os idosos assinaram o termo de consentimento livre e esclarecido, concordando em participar da pesquisa.

Para a coleta de dados foi utilizado um questionário estruturado, o qual se constituiu de questões referentes aos antecedentes pessoais, familiares e doenças autorreferidas; informações sociodemográficas; nível de atividade física, por meio do Questionário Internacional de Atividade Física - IPAQ, versão curta; variáveis comporta- 
mentais; eventos de quedas no último ano; sintomatologia depressiva usando a Escala de Depressão Geriátrica de Yersavage em versão reduzida, por meio de 15 perguntas negativas/afirmativas, na qual o resultado igual ou superior a 5 pontos estabelece o diagnóstico de depressão. Foram ainda realizadas medidas antropométricas como peso e altura, para calcular o Índice de Massa Corpórea (IMC).

\section{Diagnóstico de fragilidade (variável dependente)}

A Síndrome da Fragilidade foi identificada de acordo com os cinco critérios propostos por Fried et al. ${ }^{4}$ : 1) Perda de peso não intencional; 2) Exaustão avaliada por autorrelato de fadiga; 3) Diminuição da força de preensão manual; 4) Baixo nível de atividade física; e 5) Diminuição da velocidade de caminhada.

Perda de peso: foi definida por meio do autorrelato da perda de peso não intencional $(\geq 4,5 \mathrm{~kg}$ ou $\geq 5 \%$ do peso corporal no ano anterior), sendo computado como critério de fragilidade os idosos que relataram essa perda.

Baixa força muscular: Foi definida pelo teste de força de preensão manual (FPM) usando um dinamômetro (modelo EH101, e.clear), cujos procedimentos foram descritos previamente ${ }^{8}$. A baixa força muscular foi definida de acordo com o sexo e índice de massa corporal [IMC = massa corporal $\left.(\mathrm{kg}) / \operatorname{estatura}^{2}(\mathrm{~m})\right]$. O IMC foi classificado em três categorias ${ }^{9}:<22 \mathrm{~kg} / \mathrm{m}^{2}$, baixo peso; $22,0 \leq \mathrm{IMC} \leq 27 \mathrm{~kg} / \mathrm{m}^{2}$, adequado; $>27 \mathrm{~kg} /$ $\mathrm{m}^{2}$, sobrepeso. Para cada categoria, os pontos de corte para a FPM (kg) foi fixado no percentil 25, para homens e mulheres: baixo peso, 21,2 e 10,7 kgf; peso adequado, 24,8 e 14,6 kgf; sobrepeso 21 e 15,5 kgf, respectivamente. Foi considerado critério de fragilidade os indivíduos que apresentaram baixa força muscular corrigida pelo IMC e aqueles que foram incapazes de realizar o teste devido a limitações físicas.

Fadiga: Foi definida com base na exaustão avaliada por autorrelato de fadiga, indicado por duas questões da Center for Epidemiological Studies - Depression (CES-D $)^{10}$ : Você sentiu que teve que fazer esforço para dar conta das suas tarefas habituais? e Você não conseguiu levar adiante as suas coisas? Se o idoso respondeu na maioria das vezes e/ou sempre para uma das duas questões foi considerado como critério de fragilidade.

Lentidão no teste de caminhada: Foi definida por meio do desempenho físico no teste de caminhada de 2,44 m, cujos procedimentos foram descritos previamente ${ }^{8}$. A lentidão foi ajustada de acordo com o sexo e a altura dos idosos. A altura foi dividida em duas categorias, com base na mediana (percentil 50): homens $\leq 1,59 \mathrm{~m}$ e mulheres $\leq 1,48 \mathrm{~m}$, abaixo ou igual a mediana; homens $>1,59$ m e mulheres $>1,48 \mathrm{~m}$, acima da mediana. Para cada categoria, os pontos de corte para o teste de caminhada foram fixados no percentil 75: abaixo ou igual a mediana, $\geq 5,3$ s e $\geq 6$ s e acima da mediana, $\geq 5 \mathrm{~s} \mathrm{e} \geq 6,2 \mathrm{~s}$ (para homens e mulheres, respectivamente). Foram considerados como critério de fragilidade os idosos que pontuaram acima do ponto-corte no teste de caminhada e aqueles que foram incapazes de realizar o teste devido a limitações físicas.

Baixo nivel de atividade física: O instrumento utilizado para avaliar o nível de atividade física habitual foi o International Physical Activity Questionnaire (IPAQ), versão curta ${ }^{11}$. Os indivíduos que realizaram menos de 150 minutos por semana em atividades físicas moderadas e/ou vigorosas foram considerados insuficientemente ativos, pontuando como um critério de fragilidade.

Foram considerados "Não frágeis", os idosos que não apresentaram nenhum dos critérios; "Pré-frágeis", um ou dois dos critérios, e "Frágeis", três ou mais dos cinco critérios supracita$\operatorname{dos}^{4}$.

\section{Variáveis independentes}

Sociodemográficas: Sexo (masculino e feminino); Grupo etário (60 - 69, 70 - 79 e $\geq 80$ anos); Arranjo familiar (acompanhado e sozinho).

Comportamentais: Ingestão de bebida alcoólica (bebe, já bebeu e nunca bebeu); Uso de cigarro (fumante, ex-fumante e nunca fumou).

Condições de saúde: Índice de Massa Corporal IMC: $\left(\mathrm{IMC}<22 \mathrm{Kg} / \mathrm{m}^{2}=\right.$ peso insuficiente, 22 $\mathrm{Kg} / \mathrm{m}^{2} \leq \mathrm{IMC} \leq 27 \mathrm{Kg} / \mathrm{m}^{2}=$ adequado, e IMC $>$ $27 \mathrm{Kg} / \mathrm{m}^{2}=$ sobrepeso $^{8}$, calculado a partir dos valores da massa corporal (MC) e estatura (Est.): $\mathrm{IMC}=\mathrm{MC}(\mathrm{Kg}) /$ Est. $\left(\mathrm{m}^{2}\right)$.

Para a mensuração da MC foi utilizada uma balança digital portátil (Zhongshan Camry Eletronic, G-Tech Glass 6, China), onde o idoso permaneceu descalço e vestindo o mínimo de roupa possível. Para medir a estatura, também permaneceu descalço na posição ereta, com pés unidos e com os calcanhares, nádegas e cabeça em contato com a parede e os olhos fixos num eixo horizontal paralelo ao chão (Linha de Frankfurt). Para realizar a medida correspondente à estatura, era colocado um esquadro sobre o topo da cabe- 
ça (vértex) do examinado, formando um ângulo de $90^{\circ}$ com a parede e marcando-se esse ponto, ao final de uma inspiração.

Evento de queda no último ano: (sim e não).

Número de doenças crônicas autorreferidas: de acordo com o diagnóstico médico (sim ou não), considerando-se hipertensão e diabetes.

Sintomatologia depressiva: de acordo com a escala de depressão geriátrica de Yersavage em versão reduzida (sim e não).

Tabela 1. Distribuição das variáveis sociodemográficas, comportamentais e condições de saúde em uma amostra de idosos do município de Jequié, Bahia, Brasil, 2014.

\begin{tabular}{|c|c|c|c|}
\hline Variáveis & $\begin{array}{c}\% \\
\text { resposta }\end{array}$ & $\mathbf{N}$ & $\%$ \\
\hline $\begin{array}{l}\text { Grupo etário } \\
\text { 60-69 anos } \\
70-79 \text { anos } \\
\geq 80 \text { anos }\end{array}$ & 99,3 & $\begin{array}{l}56 \\
56 \\
26\end{array}$ & $\begin{array}{l}40,6 \\
40,6 \\
18,8\end{array}$ \\
\hline $\begin{array}{l}\text { IMC } \\
\quad<22 \\
22-27 \\
>27 \mathrm{~kg} / \mathrm{m} 2\end{array}$ & 96,4 & $\begin{array}{l}49 \\
47 \\
38\end{array}$ & $\begin{array}{l}36,5 \\
35,1 \\
28,4\end{array}$ \\
\hline $\begin{array}{l}\text { Arranjo familiar } \\
\text { Vive sozinho } \\
\text { Vive acompanhado }\end{array}$ & 100 & $\begin{array}{l}79 \\
60\end{array}$ & $\begin{array}{l}56,8 \\
43,2\end{array}$ \\
\hline $\begin{array}{l}\text { Atividade física } \\
\text { Ativo } \\
\text { Insuficientemente ativo }\end{array}$ & 98,6 & $\begin{array}{l}72 \\
65\end{array}$ & $\begin{array}{l}52,6 \\
47,4\end{array}$ \\
\hline $\begin{array}{l}\text { Tabaco } \\
\text { Fumante } \\
\text { Ex-fumante } \\
\text { Nunca fumou }\end{array}$ & 99,3 & $\begin{array}{l}14 \\
55 \\
69\end{array}$ & $\begin{array}{l}10,1 \\
39,9 \\
50,0\end{array}$ \\
\hline $\begin{array}{l}\text { Consumo de álcool } \\
\text { Bebe } \\
\text { Já bebeu } \\
\text { Nunca bebeu }\end{array}$ & 99,3 & $\begin{array}{l}19 \\
45 \\
74\end{array}$ & $\begin{array}{l}13,8 \\
32,6 \\
53,6\end{array}$ \\
\hline $\begin{array}{l}\text { Quedas } \\
\text { Sim } \\
\text { Não }\end{array}$ & 99,3 & $\begin{array}{r}38 \\
100\end{array}$ & $\begin{array}{l}27,5 \\
72,5\end{array}$ \\
\hline $\begin{array}{l}\text { Hipertensão autorreferida } \\
\text { Sim } \\
\text { Não }\end{array}$ & 100 & $\begin{array}{l}90 \\
49\end{array}$ & $\begin{array}{l}64,7 \\
35,3\end{array}$ \\
\hline $\begin{array}{l}\text { Diabetes autorreferida } \\
\text { Sim } \\
\text { Não }\end{array}$ & 100 & $\begin{array}{r}21 \\
118\end{array}$ & $\begin{array}{l}15,1 \\
84,9\end{array}$ \\
\hline $\begin{array}{l}\text { Sintomas depressivos } \\
\text { Sim } \\
\text { Não }\end{array}$ & 98,6 & $\begin{array}{l}43 \\
94\end{array}$ & $\begin{array}{l}31,4 \\
68,6\end{array}$ \\
\hline
\end{tabular}

IMC: índice de massa corporal.

\section{Análise estatística}

Foi realizada uma análise descritiva, mediante a distribuição de frequência absoluta e relativa para as variáveis categóricas e medidas de tendência central (média e mediana) e dispersão (desvio-padrão e amplitude), para as variáveis contínuas. Para análise de associação entre o perfil de fragilidade e os fatores associados foram utilizados os testes de qui-quadrado de Pearson ou exato de Fisher, com um nível de significância de 5\% ( $\mathrm{p}<0,05)$. Para a elaboração do banco de dados, foi utilizado o aplicativo Excel e, para a análise dos dados, o programa estatístico SPSS ${ }^{\circledR}$, versão 21.0

\section{Resultados}

Participaram do estudo 139 idosos, sendo 105 $(75,5 \%)$ mulheres e 34 (24,5\%) homens. A idade da população estudada variou de 60 a 101 anos, com idade média de $72,32 \pm 8,4$ anos. A idade média das mulheres foi 72,16 $\pm 8,51$ (60 - 101 anos) e dos homens foi 72,85 $\pm 8,43$ (6092 anos). Quanto à etnia, os não brancos representaram a maior parte da amostra $(85,6 \%)$. Em relação ao grau de instrução do chefe da família, $73,1 \%$ declararam serem analfabetos ou com ensino médio incompleto. As demais características estão apresentadas na Tabela 1.

A Figura 1 apresenta a prevalência dos idosos não frágeis, pré-frágeis e frágeis. As análises do perfil de fragilidade foram feitas com 136 idosos,

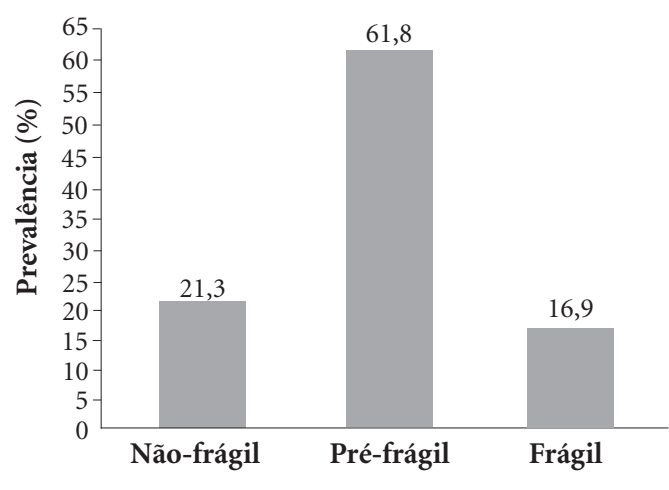

Figura 1. Prevalência do perfil de fragilidade em uma amostra de idosos do município de Jequié, Bahia, Brasil, 2014. 
o que equivale a $97,8 \%$ das respostas desta variável. Observou-se uma alta prevalência de idosos pré-frágeis $(61,8 \%)$ e de idosos frágeis $(16,9 \%)$.

A Tabela 2 apresenta associação do perfil de fragilidade com as características sociodemográficas, sexo, IMC, atividade física, variáveis comportamentais, quedas, doenças autorreferidas e sintomas depressivos. Observou-se uma associação do perfil de fragilidade com o grupo etário $(\mathrm{p}<0,001)$, quanto mais idoso, maior a probabilidade de ser pré-frágil e frágil. Foi encontrada uma maior prevalência de idosos pré-frágeis $(64,7 \%)$ e frágeis $(18,6 \%)$ no sexo feminino. Também houve associação do perfil de fragilidade com IMC ( $\mathrm{p}=0,018$ ), observando uma maior prevalência dos idosos frágeis $(27,1 \%)$ com IMC $<22 \mathrm{~kg} / \mathrm{m}^{2}$ (baixo peso); com o arranjo familiar $(\mathrm{p}=0,014)$, obtendo uma maior prevalência de fragilidade $(23,4 \%)$ nos idosos que vivem sozinhos; com atividade física $\mathrm{p}<0,001$ (insuficientemente ativo), e número de quedas $(\mathrm{p}=0,043)$, observando uma prevalência de $(28,9 \%)$ dos idosos frágeis caidores. As demais associações estão apresentadas na Tabela 2.

Tabela 2. Perfil de fragilidade e fatores associados em uma amostra de idosos do município de Jequié, Bahia, Brasil, 2014.

\begin{tabular}{|c|c|c|c|c|c|}
\hline \multirow[b]{2}{*}{ Variáveis } & \multicolumn{3}{|c|}{ Diagnóstico de fragilidade } & \multirow[b]{2}{*}{ Total } & \multirow[b]{2}{*}{ P-valor } \\
\hline & Não-frágil & Pré-frágil & Frágil & & \\
\hline \multicolumn{3}{|l|}{ Grupo etário } & \multirow{4}{*}{$\begin{array}{r}9(16,4 \%) \\
3(5,5 \%) \\
11(42,3 \%)\end{array}$} & 55 & \multirow[t]{4}{*}{$<0,001$} \\
\hline 60-69 anos & $7(12,7 \%)$ & \multirow{2}{*}{$\begin{array}{r}39(70,9 \%) \\
33(60 \%)\end{array}$} & & \multirow{2}{*}{55} & \\
\hline 70-79 anos & $19(34,5 \%)$ & & & & \\
\hline$\geq 80$ anos & $3(11,5 \%)$ & $12(46,2 \%)$ & & & \\
\hline \multicolumn{3}{|l|}{ Sexo } & \multirow{3}{*}{$\begin{array}{r}4(11,8 \%) \\
19(18,6 \%)\end{array}$} & \multirow{3}{*}{$\begin{array}{r}34 \\
102\end{array}$} & \multirow[t]{3}{*}{0,066} \\
\hline Masculino & $12(35,3 \%)$ & $18(52,9 \%)$ & & & \\
\hline Feminino & $17(16,7 \%)$ & $66(64,7 \%)$ & & & \\
\hline \multicolumn{3}{|l|}{ IMC } & \multirow{4}{*}{$\begin{array}{r}13(27,1 \%) \\
7(15,2 \%) \\
3(7,9 \%)\end{array}$} & \multirow{2}{*}{48} & \multirow[t]{4}{*}{0,018} \\
\hline$<22$ & $13(27,1 \%)$ & $22(45,8 \%)$ & & & \\
\hline $22-27$ & $10(21,7 \%)$ & $29(63 \%)$ & & & \\
\hline$>27 \mathrm{~kg} / \mathrm{m} 2$ & $4(10,5 \%)$ & $31(81,6 \%)$ & & & \\
\hline \multicolumn{3}{|l|}{ Arranjo familiar } & \multirow{3}{*}{$\begin{array}{r}18(23,4 \%) \\
5(8,5 \%)\end{array}$} & \multirow{3}{*}{$\begin{array}{l}77 \\
59\end{array}$} & \multirow[t]{3}{*}{0,014} \\
\hline Vive sozinho & $11(14,3 \%)$ & $48(62,3 \%)$ & & & \\
\hline Vive acompanhado & $18(30,5 \%)$ & $36(61 \%)$ & & & \\
\hline \multicolumn{3}{|l|}{ Atividade física } & \multirow{3}{*}{$\begin{array}{l}11(15,3 \%) \\
11(17,5 \%)\end{array}$} & \multirow{3}{*}{$\begin{array}{l}72 \\
63\end{array}$} & \multirow[t]{3}{*}{$<0,001$} \\
\hline Ativo & $29(40,3 \%)$ & $32(44,4 \%)$ & & & \\
\hline Insuficientemente ativo & $0(0,0 \%)$ & $52(82,5 \%)$ & & & \\
\hline \multicolumn{3}{|l|}{ Tabaco } & $3(21,4 \%)$ & 14 & 0,347 \\
\hline Fumante & $4(28,6 \%)$ & $7(50 \%)$ & $10(18,2 \%)$ & 55 & \\
\hline Ex-fumante & $15(27,3 \%)$ & $30(54,5 \%)$ & $10(14,9 \%)$ & 67 & \\
\hline Nunca fumou & $10(14,9 \%)$ & $47(70,1 \%)$ & & & \\
\hline Consumo de álcool & & & $4(21,1 \%)$ & 19 & 0,515 \\
\hline Bebe & $3(15,8 \%)$ & $12(63,2 \%)$ & $5(11,1 \%)$ & 45 & \\
\hline Já bebeu & $13(28,9 \%)$ & $27(60 \%)$ & $14(19,4 \%)$ & 72 & \\
\hline Nunca bebeu & $13(18,1 \%)$ & $45(62,5 \%)$ & & & \\
\hline Quedas & & & $11(28,9 \%)$ & 38 & 0,043 \\
\hline $\operatorname{Sim}$ & $5(13,2 \%)$ & $22(57,9 \%)$ & $12(12,2 \%)$ & 98 & \\
\hline Não & $24(24,5 \%)$ & $62(63,3 \%)$ & & & \\
\hline Hipertensão autorreferida & & & $16(18,4 \%)$ & 87 & 0,603 \\
\hline Sim & $20(23 \%)$ & $51(58,6 \%)$ & $7(14,3 \%)$ & 49 & \\
\hline Não & $9(18,4 \%)$ & $33(67,3 \%)$ & & & \\
\hline Diabetes autorreferida & & & $2(9,5 \%)$ & 21 & 0,330 \\
\hline Sim & $3(14,3 \%)$ & $16(76,2 \%)$ & $21(18,3 \%)$ & 115 & \\
\hline Não & $26(22,6 \%)$ & $68(59,1 \%)$ & & & \\
\hline Sintomas depressivos & & & $10(23,3 \%)$ & 43 & 0,086 \\
\hline $\operatorname{Sim}$ & $12(27,9 \%)$ & $21(48,8 \%)$ & $12(13 \%)$ & 92 & \\
\hline Não & $17(18,5 \%)$ & $63(68,5 \%)$ & & & \\
\hline
\end{tabular}

IMC: Índice de massa corporal. 


\section{Discussão}

No presente estudo, a prevalência de idosos frágeis foi de $16,9 \%$, pré-frágil $61,8 \%$, e não frágil $21,3 \%$. A taxa de fragilidade foi superior a observada na literatura internacional por Fried et al. $^{4}$, que encontrou uma prevalência de $6,9 \%$, e também na literatura nacional, por Vieira et al. ${ }^{5}$, $8,7 \%$, e Neri et al. ${ }^{12}, 9,1 \%$.

No entanto, os resultados deste estudo se assemelham ao observado em uma comunidade da região nordeste que encontrou uma prevalência de $17,1 \%$ de fragilidade e $60,1 \%$ de pré-fragilida$\mathrm{de}^{13}$, o que reforça a influência das diversidades regionais, visto que as características do contexto social afetam a saúde, gerando desigualdades nas exposições e vulnerabilidades ${ }^{14}$.

Observou-se ainda a supremacia de idosos na condição de pré-fragilidade, corroborando com estudos citados anteriormente $e^{4-6,12,13}$, que aponta para a importância e a necessidade de intervenções visando prevenir o agravamento da condição de pré-fragilidade para um quadro de fragilidade, evitando, assim, os desfechos adversos melhorando a qualidade de vida dos idosos.

Vale ressaltar que ainda são escassas as informações sobre a prevalência e incidência da síndrome de fragilidade em idosos, possivelmente devido à falta de consenso sobre os critérios que possam ser utilizados como instrumento de triagem em diferentes populações ${ }^{15}$. No presente estudo, houve predomínio de fragilidade no sexo feminino, e esta condição esteve relacionada com o envelhecimento, esses resultados corroboram com outros estudos ${ }^{4,16}$. No entanto, Remor et al. ${ }^{6}$ não encontraram diferenças significativas no perfil de fragilidade no grupo etário de 60 a 79 anos e idosos longevos (maiores de 80 anos).

Com relação à elevada prevalência da síndrome na população feminina, ressalta-se que as mulheres estão associadas a uma fragilidade intrínseca, pelo fato de terem menor massa magra e força muscular em relação aos homens e, também, por serem mais vulneráveis aos efeitos extrínsecos da fragilidade como sarcopenia ${ }^{4}$. Além do mais, o fato das mulheres viverem mais que os homens aumentam sua predisposição às doenças crônicas, visto que, no processo de envelhecimento, as próprias modificações fisiológicas e funcionais tornam o idoso mais susceptível a tais doenças ${ }^{17}$, como observado por outros autores ${ }^{4,5}$.

No presente estudo, não houve associação significativa entre fragilidade e doenças crônicas, assim como no estudo de Miguel et al. ${ }^{18}$, o que aponta para a necessidade de outras investiga- ções para esclarecer essa relação. A presença de comorbidades nem sempre é a acompanhada de fragilidade, no entanto, estas podem aumentar o risco de eventos adversos à saúde ${ }^{19}$, tornando o idoso mais vulnerável a fragilidade pelas limitações físicas que podem surgir com o aparecimento das doenças ${ }^{6}$.

No estudo de Vieira et al. ${ }^{5}$ foi encontrada associação entre sintomas depressivos e fragilidade, entretanto, no presente estudo não foi evidenciada essa associação.

Outro aspecto importante e descrito na literatura é a fragilidade como um importante fator de risco para quedas ${ }^{2}$. No presente estudo foi verificada associação entre quedas e fragilidade, corroborando com outros autores ${ }^{4,20}$. A explicação para essa associação ainda não é bem conhecida, porém como a fragilidade aumenta gradativamente com a idade, e o envelhecimento leva a uma redução da acuidade visual, do equilíbrio corporal, mobilidade e função física, refere-se que estes possam explicar a ocorrência de quedas nos idosos frágeis ${ }^{21}$. Esse resultado pode também ser explicado pela associação existente entre queda e os componentes da síndrome, principalmente a sarcopenia, que isoladamente prenuncia queda ${ }^{22}$.

Em um estudo realizado por Vieira et al. ${ }^{5}$, os autores identificaram que o baixo nível de atividade física e a lentidão na velocidade de marcha foram os dois critérios de fragilidade mais frequentes entre os idosos pré-frágeis e frágeis, e constataram também, que os idosos frágeis apresentavam limitações nas atividades instrumentais da vida diária e restrição nas atividades avançadas. No atual estudo, foi encontrada uma associação entre baixo nível de atividade física e fragilidade, fazendo-se presente em 17,5\% dos idosos frágeis. Esta associação pode ser explicada pela relação entre sedentarismo e a baixa força de preensão e lentidão de marcha ${ }^{23}$, que compõem os critérios utilizados para diagnosticar a síndrome. Acrescenta-se ainda, que, aos aspectos da fragilidade, incluem-se a redução na mobilidade, fraqueza muscular, marcha anormal, menor tolerância ao exercício, instabilidade de equilíbrio, má nutrição e sarcopenia ${ }^{4,24}$, que poderiam levar os idosos à redução das práticas de atividades físicas.

Quanto ao IMC, houve associação significativa com o perfil de fragilidade, sendo o baixo peso prevalente nos idosos frágeis. Esses resultados corroboram com o estudo de Moretto et al. ${ }^{25}$. No entanto, a literatura também aponta associação entre fragilidade e obesidade ${ }^{18,26}$. Os problemas nutricionais que afetam a população idosa podem antecipar o desenvolvimento da fragilidade 
e vulnerabilidades, assim, tanto a desnutrição quanto o sobrepeso podem ser considerados como marcadores potenciais da síndrome, levando em consideração as possíveis complicações que podem acarretar na vida dos idosos ${ }^{27}$.

Com relação ao arranjo familiar, foi evidenciado que a prevalência de fragilidade foi maior nos idosos que residiam sozinhos, resultados consistentes com o de Vieira et al. ${ }^{5}$, que verificaram um aumento gradativo da condição de fragilidade entre os idosos que não possuíam parceiros fixos, evidenciando a importância do suporte social na proteção dos eventos desfavoráveis à saúde do idoso ${ }^{28}$. Contudo, Amaral et al. ${ }^{29}$, ao buscarem analisar a associação entre o apoio social e a fragilidade em idosos, observaram que não houve associação da fragilidade com as variáveis de apoio social, exceção feita apenas na modalidade tarefa doméstica.

Assim, faz-se presente a necessidade de atuação e desenvolvimento de pesquisas pelos profissionais de saúde frente a esta síndrome, propondo a execução de políticas de saúde com medidas preventivas e de controle. Tais medidas visam uma intervenção mais eficaz na realidade em questão, que é caracterizada por grande número de doenças crônicas, quedas, fraturas, perda da autonomia e dependência, o que leva a aumento das taxas de hospitalização.

\section{Conclusão}

Neste estudo, observou-se uma alta prevalência de idosos pré-frágeis e frágeis e identificou fatores associados a esta síndrome. Foi observada associação entre perfil de fragilidade e grupo etário, IMC, arranjo familiar, atividade física e número de quedas.

As condições de fragilidade e especialmente a pré-fragilidade devem ser destacadas na realização de medidas preventivas e intervencionistas para retardar e atenuar o declínio funcional, visando à promoção do envelhecimento ativo para melhorar a qualidade de vida dos mesmos.

Uma das possíveis limitações deste estudo poderia estar relacionada a eventual possibilidade de coexistirem situações de fragilidade que não foram captadas pelo método utilizado neste trabalho. Uma vez que, por se tratar de uma população idosa e com baixa classe econômica, possivelmente o viés da memória pode estar contribuindo para a baixa qualidade das respostas.

\section{Colaboradores}

PHS Santos e JAO Carneiro participaram da elaboração e desenho do projeto, e também da coleta, análise e interpretação dos dados. MH Fernandes, CA Cassotti e RS Coqueiro, colaboraram na redação final e revisão crítica do artigo.

\section{Agradecimentos}

Ao Programa de Educação pelo Trabalho para Saúde (PET- Saúde), Grupo Idoso I, pelo apoio e colaboração. 
Referências

1. Lourenço RA, Martins CSF, Sanchez MAS, Veras RP. Assistência ambulatorial geriátrica: hierarquização da demanda. Rev Saude Publica 2005; 39(2):311-318.

2. Fabrício SCC, Rodrigues RAP. Revisão da literatura sobre fragilidade e sua relação com o Envelhecimento. Rev. RENE 2008; 9(2):113-119.

3. Ferriolli E, Morigute JC, Formighieri PF. O idoso frágil. In: Lopes AC, Neto VA, organizadores. Tratado de Clínica Médica. São Paulo: Roca Editora; 2006. p. 4454-4460.

4. Fried LP, Tangen CM, Walston J, Newman AB, Hirsch C, Gottdiener J, Seeman T, Tracy R, Kop WJ, Burke G, Mc Burnie MA. Frailty in older adults: evidence for a phenotype. J Gerontol A Biol Sci Med Sci 2001; 56(3):M146-M156.

5. Vieira RA, Guerra RO, Giacomin KC, Vasconcelos KSS Andrade ACS, Pereira LSM, Dias JMD, Dias RC. Prevalência de fragilidade e fatores associados em idosos comunitários de Belo Horizonte, Minas Gerais, Brasil: dados do Estudo FIBRA. Cad Saude Publica 2013; 29(8):1631-1643.

6. Remor CB, Bós AJG, Werlang MC. Características relacionadas ao perfil de fragilidade no idoso. Sci Med. 2011;21(3):107-112

7. Brasil. Ministério da Saúde (MS). Conselho Nacional de Saúde. Resolução no 466, de 12 de dezembro de 2012. Diário Oficial da União 2013; $13 \mathrm{dez.}$

8. Santos KT, Fernandes MH, Reis LA, Coqueiro RS, Rocha SV. Depressive symptoms and motor performance in the elderly: a population based study. Rev Bras Fisioter 2012; 16(4):295-300.

9. American Academy of Family Physicians, American Dietetic Association, National Council on the Aging. Nutrition screening e intervention resources for healthcare professionals working with older adults. Nutrition Screening Initiative. Washington: American Dietetic Association; 2002. [acessado 2010 abr 7]. Disponível em: http://www.eatright.org/cps/rde/xchg/ada/hs.xsl/ nutrition_nsi enu html.htm

10. Batistoni SST, Neri AL, Cupertino APFB. Validade da escala de depressão do Center for Epidemiological Studies entre idosos brasileiros. Rev Saude Publica 2007; 41(4):598-605.

11. Craig CL, Marshall AL, Sjostrom M, Bauman AE, Booth ML, Ainsworth BE, Prati M, Ekelund U, Yngve A, Sallis JF, Oja P. International Physical Activity Questionnaire: 12-country reliability and validity. Med Sci Sports Exerc 2003; 35(8):1381-1395.

12. Neri AL, Yassunda MS, Araújo LF, Eulálio MC, Cabra BE, Siqueira MEC, Santos GA, Moura JGA. Metodologia e perfil sociodemográfico, cognitivo e de fragilidade de idosos comunitários de sete cidades brasileiras: Estudo FIBRA. Cad Saude Publica 2013; 29(4):778-792.

13. Souza AC, Dias RC, Maciel AC, Guerra RO. Frailty syndrome and associated factors in community-dewelling elderly in Northeast Brasil. Arch Gerontol Geriatr 2012; 54(2):95-101.

14. Geib LTC. Determinantes sociais da saúde do idoso. Cien Saude Colet 2012; 17(1):123-133.

15. Macedo C, Gazzola JM, Najas M. Síndrome da fragilidade no idoso: importância da fisioterapia. Arq Bras ciênc saúde 2008; 33(3):177-184.

16. Chen CY, Wu SC, Chen LJ, Lue BH. The prevalence of subjective frailty and factors associated with frailty in Taiwan. Arch Gerontol Geriatr 2010; 50(Supl. 1):43-47.
17. Oliveira SMJV, Santos JLF, Lebrão ML, Duarte YAO, Pierin AMG. Hipertensão arterial referida em mulheres idosas: Prevalência e fatores associados. Texto Contexto Enferm 2008; 17(2):241-249.

18. Miguel RCC, Dias RC, Dias JMD, Silva SLA, Filho PRM, Ribeiro TM. Síndrome da fragilidade no idoso comunitário com osteoartrite. Rev Bras Reumatol 2012; 52(3):331-347.

19. Fried LP, Walston J. Frailty and failure to thrive. In: Hazzard WR, Tinetti M, Halter J, Blass J, Ouslander J. Principles of geriatric medicine and gerontology. $5^{\text {th }} \mathrm{ed}$. Nova York: Mac Graw-Hill; 2003. p. 1487-502.

20. Woods NF, La Croix AZ, Gray SL, Aragaki A, Cochrane BB, Brunner RL, Masaki K, Murray A. Newman AB. Frailty: emergence and consequences in women aged 65 and older in the Women's Health Initiative Observational Study. J Am Geriatr Soc 2005; 53(8):1321-1330.

21. Guimarães JMN, Farinatti PTV. Análise descritiva de variáveis teoricamente associadas ao risco de quedas em mulheres idosas. Rev Bras Med Esporte 2005; 11(5):299-305.

22. Gill TM, Gahbauer EA, Han L, Allore HG. The relationship between intervening hospitalizations and transitions between frailty states. J Gerontol A Biol Sci Med Sci 2011; 66(11):1238-1243.

23. Costa TB, Neri AL. Medidas de atividade física e fragilidade em idosos: dados do FIBRA Campinas, São Paulo, Brasil. Cad Saude Publica. 2011; 27(8):1537-1550.

24. Walston J, Hadley EC, Ferrucci L, Guralnik JM, Newman AB, Studenski SA, Ershler WB, Harris T, Fried LP. Research agenda for frailty in older adults: toward a better understanding of physiology and etiology: summary from the American geriatrics society/national institute on aging research conference on frailty in older adults. J Am Geriatr Soc 2006; 54(6):991-1001.

25. Moretto MC, Alves RM, Neri AL, Guariento ME. Relationship between nutritional status and frailty in Brazilian elderly. Rev Bras Clin Med 2012; 10(4):267-271.

26. Blaum CS, Xue QL, Michelon E, Semba RD, Fried LP. The association between obesity and the frailty syndrome in older women: the Women's Health and Aging Studies. J Am Geriatr Soc 2005; 53(6):927-934.

27. Topinkova E. Aging, disability and frailty. Ann Nutr Metab 2008; 52(Supl. 1):6-11

28. d'Orsi E, Xavier AJ, Ramos LR. Trabalho, suporte social e lazer protegem idosos da perda funcional: Estudo Epidoso. Rev Saude Publica 2011; 45(4):685-692.

29. Amaral FLJS, Guerra RO, Nascimento AFF, Maciel ACC. Apoio social e síndrome da fragilidade em idosos residentes na comunidade. Cien Saude Colet 2013; 18(6):1835-1846.

Artigo apresentado em 14/06/2014

Aprovado em 05/11/2014

Versão final apresentada em 07/11/2014 